\title{
Beclin-1 expression serves as an important biomarker for carcinogenesis and evolution in lung adenocarcinoma presenting as ground glass opacity
}

\author{
Ling Chen ${ }^{1 \#}$, Hailei Du ${ }^{2 \#}$, Fangxiu Luo ${ }^{3}$, Xueyu Chen ${ }^{4}$, Yong Li ${ }^{1}$, Qijian Cheng ${ }^{1}$ \\ ${ }^{1}$ Department of Respiratory Medicine, Ruijin Hospital North, Shanghai Jiaotong University School of Medicine, Shanghai, China; ${ }^{2}$ Department of \\ Thoracic Surgery, Ruijin Hospital, Shanghai Jiaotong University School of Medicine, Shanghai, China; ${ }^{3}$ Department of Pathology, ${ }^{4}$ Department of \\ Thoracic Surgery, Ruijin Hospital North, Shanghai Jiaotong University School of Medicine, Shanghai, China \\ Contributions: (I) Conception and design: Q Cheng; (II) Administrative support: F Luo; (III) Provision of study materials or patients: L Chen, H Du; \\ (IV) Collection and assembly of data: L Chen, H Du, X Chen; (V) Data analysis and interpretation: Y Li; (VI) Manuscript writing: All authors; (VII) \\ Final approval of manuscript: All authors. \\ \#These authors contributed equally to this work. \\ Correspondence to: Qijian Cheng. No. 999, Xi Wang Road, Jiading New Town Center, Jiading District, Shanghai, China. \\ Email: chengqijian@aliyun.com.
}

Background: The prognostic impact of Beclin-1 and relationship between Beclin-1 expression and carcinogenesis of lung adenocarcinoma has not been clarified.

Methods: The data of 10 patients with atypical adenomatoid hyperplasia (AAH), 20 patients with adenocarcinoma in situ (AIS), 28 patients with minimally invasive adenocarcinoma (MIA), and 50 patients with invasive adenocarcinoma (IA) who underwent surgical resection, were retrospectively reviewed. Normal lung tissues (NTs) were also collected for comparison. The expression levels of Beclin-1 mRNA and protein were detected by RT-PCR and western blotting, respectively. Immunohistochemistry was also performed.

Results: The transcriptional expression of Beclin-1 in lung adenocarcinoma presenting as GGO was significantly lower than that in NTs $(\mathrm{P}<0.05)$, and patients with MIA and IA showed a lower expression of Beclin-1 than that in patients with AAH and AIS $(\mathrm{P}<0.01)$. Lung adenocarcinomas with low expression of Beclin-1 were in more advanced stage (stage 0-I vs. stageII_III: $0.24 \pm 0.11$ vs. $0.17 \pm 0.03, \mathrm{P}<0.01$ ), had more lymph node metastasis $(\mathrm{P}<0.01)$, and were of more invasive pathological subtype $(\mathrm{P}<0.01)$ than lung adenocarcinomas with high expression of Beclin-1. Low expression of Beclin-1 predicted worse survival in patients with IA $(\mathrm{P}<0.05)$.

Conclusions: Beclin-1 expression was related with the evolution of lung adenocarcinoma. Beclin-1 may be an important marker for predicting the prognosis of patients with lung adenocarcinoma manifested as GGO.

Keywords: Autophagy; Beclin-1; lung adenocarcinoma; prognosis

Submitted Feb 13, 2020. Accepted for publication Jun 05, 2020.

doi: $10.21037 /$ tcr-20-1001

View this article at: http://dx.doi.org/10.21037/tcr-20-1001

\section{Introduction}

Modern radiological techniques, especially computed tomography (CT), can aid the detection of more earlystage lung cancer. On CT images, early-stage lung cancer is often displayed as ground glass opacity (GGO). According to the appearance or absence of solid components in the lesion, GGO lesions can be classified into pure GGO (pGGO) and mixed GGO (mGGO). Meanwhile, mixed GGO could be further divided into GGO-predominant and solid-predominant. The pathologies of malignant GGO are commonly associated with lung adenocarcinoma (1). In 2011, the International Association for the Study of Lung 
Cancer (IASLC), the American Thoracic Society (ATS), and the European Respiratory Society (ERS) proposed an International Multidisciplinary Lung Adenocarcinoma Classification (2). There are five subtypes of invasive adenocarcinomas (IAs), including acinar, lepidic, papillary, micropapillary, and solid predominant. Adenocarcinomas with a predominant lepidic growth pattern and an invasive component size $>5 \mathrm{~mm}$ were classified as lepidic predominant adenocarcinomas (LPAs). Patients with adenocarcinoma in situ (AIS) and minimally invasive adenocarcinoma (MIA) had an almost $100 \%$ diseasespecific survival with complete surgical resection compared to that in patients with stage I adenocarcinoma with $30 \%$ recurrence rate (3). Patients with LPA had significantly better prognosis than patients with other IAs. Therefore, the lepidic component is regarded as a precursor of an invasive tumor. Several studies have demonstrated AIS as a precursor of IA. Considering the dramatic differences in the clinical outcome across this spectrum of lung adenocarcinomas, it is obvious that these newly defined pathological intermediates represent clinically relevant entities and present an opportunity to understand events in disease progression.

Autophagy is considered as a cellular catabolic process, and it exerts an essential role in the degradation of cellular components in lysosomes or recycles long-lived proteins and organelles (4). Autophagy plays a dual role in malignant tumors, as both a suppressor and a promoter, depending on the context the cancer cells stay in. Thus, the modification of autophagy has potential value in cancer therapy $(5,6)$. Beclin-1, the firstly identified mammalian autophagyrelated gene or protein, plays a vital role in the initiation and the regulation of autophagy. Previous publications demonstrated that defects in autophagy exerts an important role in tumorigenesis and tumor progression (7). Moreover, the decreased expression of Beclin-1 has been reported to be correlated to the primary tumor growth in NSCLC (8). The carcinogenesis and evolution of lung cancer is a multistep changing process. However, whether Beclin-1 has role in the progression of lung adenocarcinoma presenting as GGO is not yet confirmed. Therefore, to assess the role of autophagy in lung adenocarcinoma, we detected the expression of Beclin-1 in 108 patients with lung preinvasive and invasive lesions, and analyzed its relationship with the patients' clinicopathological factors. The present study aims to (I) determine the changes in the expression levels of autophagy genes and proteins involving in the occurrence and development of lung lesions in patients with atypical adenomatoid hyperplasia (AAH), AIS, MIA, and IA ; (II) investigate the potential role of autophagy in lung adenocarcinoma presenting as GGO. We present the following article/case in accordance with the REMARK reporting checklist (available at http://dx.doi.org/10.21037/ tcr-20-1001).

\section{Methods}

\section{Patients and samples}

We retrospectively evaluated the data of a series of patients with surgically resected lung lesions in Ruijin Hospital North Affiliated to Shanghai Jiaotong University School of Medicine recorded from January 2014 to December 2015. Patients with GGO lesion $(\leq 3 \mathrm{~cm})$ in lung and pathologically confirmed AAH, AIS, MIA, and IA were included. Patients with induction therapy, with positive surgical margins, or with concurrent disease progression, were excluded. Each specimen was collected from a lesion and from the normal lung tissue (NT) $(>5 \mathrm{~cm}$ from the edge of the tumor). The resected tissue specimens were washed with physiological saline and immediately stored in a liquid nitrogen tank. Demographic information of the patients was collected. Data pertaining to clinical variables and followup information were gathered by reviewing the patients' medical records. Tumors were classified based on the 2011 IASLC/ATS/ERS classification and the 2015 WHO classification. The study was conducted in accordance with the Declaration of Helsinki (as revised in 2013). The study was approved by Ethics Committee of Ruijin North Hospital (No.2017139) Ruijin Hospital North affiliated Shanghai Jiaotong University School of Medicine approved this retrospective biomarker study. and informed consent was taken from all the patients.

\section{Measurement of Beclin-1 mRNA and protein expression}

Each frozen specimen was cut into 1-mm3 fragments and then grinded. RNA was extracted using Trizol reagent. The concentration and purity of RNA were determined at $260 / 280 \mathrm{~nm}$. Beclin- 1 primer sequences were as follows: the sense strand was 5'-AGCTGCCGTTATACTGTTCTG-3', and the antisense strand was 5'-ACTGCCTCCTGTGTCTTCAATCTT-3'. The internal reference gene GAPDH primer sequences were as follows: the sense strand was 
5'-GAAGGTGAAGGTCGGAGTC-3', and the antisense strand was 5'-GAAGATGGTGATGGGATTTC-3' [all synthesized by Sangon Biotech (Shanghai) Co., Ltd.]. The PCR conditions were as follows: $94{ }^{\circ} \mathrm{C}$ for $1 \mathrm{~min}, 55^{\circ} \mathrm{C}$ for $1.5 \mathrm{~min}$, and $72{ }^{\circ} \mathrm{C}$ for $5 \mathrm{~min}$, with a total of 30 cycles. The results were analyzed by Quanlityone, and the ratio of the positive band to the optical density of GAPDH was used as the relative expression.

Cells were lysed in the radioimmunoprecipitation assay buffer, and the lysates were collected after high-speed centrifugation. The protein concentration was determined using a BCA kit (abcam), and proteins were isolated by $12 \%$ sodium dodecyl sulfate-polyacrylamide gel electrophoresis and then transferred to a $0.2-\mu \mathrm{m}$ polyvinylidene fluoride membrane. The membrane was blocked overnight in $5 \%$ skim milk with Tris-buffered saline containing Tween-20 and then incubated overnight with antibodies for Beclin-1 (Santa Cruz Biotechnology), or $\beta$-actin (Santa Cruz Biotechnology) at $4{ }^{\circ} \mathrm{C}$. Next, the membrane was incubated with secondary antibodies at room temperature for 60 min. The protein bands were observed on X-ray films after incubation with ECL reagents.

Beclin-1 expression was also evaluated by immunohistochemical staining. Briefly, anti-Beclin-1 mouse monoclonal antibody (Santa Cruz Biotechnology) was diluted at 1:100. Two pathologists reviewed the immunohistochemical sections, performed double-blind scoring, randomly selected five $400 \times$ fields, counting 100 cells in each field, and controversies were discussed to obtain the score with consensus. The scoring criteria were as follows: score A: positive cells comprising $\leq 10 \%$ were counted as 1 point; positive cells comprising $11-50 \%$ were counted as 2 points; positive cells comprising $51-75 \%$ were counted as 3 points; and positive cells comprising $>75 \%$ were counted as 4 points. Staining intensity was observed under low magnification $(100 \times)$ and scored as follows: score B: 0 indicated no staining; 1 implied light yellow staining; 2 meant brownish yellow staining; and 3 indicated brownish staining; the final score was calculated as $\mathrm{A} \times \mathrm{B}$. Low expression was defined as a score $\leq 3$, and high expression was defined as a score $>3$.

\section{Study endpoint}

The endpoint of our study was overall survival (OS) that defined as survival from the date of surgery to the date of death due to cancer. All patients were followed up to
January 2019. The median follow up time is 49 months, range, 37-60 months.

\section{Statistical analysis}

Grayscale values were read in Photoshop CS2.0 (Adobe, San Jose, CA, USA). Data of multiple groups were compared by one-way analysis of variance. The chi-square test was applied to compare the clinical factors between low-expression and high-expression groups. The unpaired Student's $t$-test was carried out when continuous variables were normally distributed, and if not, the Wilcoxon rank sum test was used. The Cox proportional hazards model was fit to identify the significant prognostic factors for survival using SPSS Statistics 21 (IBM Inc., Armonk, New York). Overall survival (OS) was estimated using the KaplanMeier method and compared by log-rank test across the different groups. The date of surgical resection was set as the starting point, and the date of death or survival followup was set as the endpoint of OS. Continuous variables that were normally distributed were summarized using mean values and $\mathrm{SD}$, and those that were not normally distributed were summarized using median and interquartile range. Differences were considered to be statistically significant when the $\mathrm{P}$ value was $<0.05$.

\section{Results}

\section{Baseline characteristics}

Of the 108 patients, 30 were males and 78 were females. Their mean age was 56.3 years, and $86(80 \%)$ patients with lung adenocarcinoma were in stage I. A total of 12 (11.1\%) patients were confirmed to have lymph node metastasis. In terms of the pathological subtype, there were $10(9 \%)$ patients with AAH, 20 (19\%) patients with AIS, 28 (26\%) patients with MIA, and 50 (46\%) patients with IA, consisting of 12 patients with LPA, 20 patients with acinar predominant adenocarcinoma, 6 patients with papillary predominant adenocarcinoma, 8 patients with micropapillary predominant adenocarcinoma, and 4 patients with solid predominant adenocarcinoma (Table 1).

\section{Relationship between Beclin-1 expression and invasiveness of lung adenocarcinoma}

The expression of Beclin-1 was not associated with sex $(\mathrm{P}>0.05)$, smoking $(\mathrm{P}>0.05)$, and age $(\mathrm{P}>0.05)$. Regarding 
Table 1 Clinicopathological features of lung adenocarcinoma based on high or low Beclin-1 expression

\begin{tabular}{|c|c|c|c|c|}
\hline \multirow{2}{*}{ Variables } & \multirow{2}{*}{ Total number - } & \multicolumn{2}{|c|}{ Beclin 1 expression } & \multirow{2}{*}{$\mathrm{P}$} \\
\hline & & High & Low & \\
\hline $\mathrm{AAH}, \mathrm{AIS}$ & 30 & 22 & 8 & $<0.01$ \\
\hline MIA, IA & 78 & 32 & 46 & \\
\hline Sex & & & & $>0.05$ \\
\hline Male & 30 & 18 & 12 & \\
\hline Female & 78 & 36 & 42 & \\
\hline Smoking & & & & $>0.05$ \\
\hline Yes & 34 & 20 & 14 & \\
\hline No & 74 & 34 & 40 & \\
\hline Age, years & & & & $>0.05$ \\
\hline$<60$ & 48 & 28 & 20 & \\
\hline$>60$ & 60 & 26 & 34 & \\
\hline Stage & & & & $<0.01$ \\
\hline $0-1$ & 86 & 52 & 34 & \\
\hline II-III & 12 & 2 & 10 & \\
\hline $\begin{array}{l}\text { Nodal } \\
\text { involvement }\end{array}$ & & & & $<0.01$ \\
\hline NO & 86 & 52 & 34 & \\
\hline $\mathrm{N} 1 / 2$ & 12 & 2 & 10 & \\
\hline $\begin{array}{l}\text { Pathologic } \\
\text { subtype }\end{array}$ & 50 & & & $<0.01$ \\
\hline Acinar & 20 & 4 & 16 & \\
\hline Lepidic & 12 & 6 & 6 & \\
\hline Papillary & 6 & 1 & 5 & \\
\hline Micropapillary & 8 & 0 & 8 & \\
\hline Solid & 4 & 1 & 3 & \\
\hline
\end{tabular}

NT, lung normal tissues; $A A H$, atypical adenomatous hyperplasia; AIS, adenocarcinoma in situ; MIA, minimally invasive adenocarcinoma; IA, invasive adenocarcinoma.

the invasiveness of lung adenocarcinoma, there were more patients with AAH and AIS who had high Beclin-1 expression $(22,73 \%)$. Compared with patients with MIA and IA, only 32 (41\%) patients had high Beclin-1 expression $(\mathrm{P}<0.01)$. Patients with early-stage $(0-\mathrm{I})$ lung adenocarcinoma showed a less incidence of low Beclin-1 expression compared to those with advanced stages (II-III). In stage I, 34 (40\%) patients showed low Beclin-1 expression compared to 10 (83\%) patients in stage II-III. Low Beclin-1 expression was also associated with lymph node metastasis (node-negative: $40 \%$, node-positive: $60 \%$; $\mathrm{P}<0.01$ ). Patients with the highest invasive subtype (micropapillary and solid predominant type) showed low Beclin-1 expression. Especially, all patients with the micropapillary predominant subtype exhibited low Beclin-1 expression (Table 1). Further analysis indicated that Beclin-1 was an independent risk factor related to overall survival for patients with lung adenocarcinoma presenting as GGO (hazard ratio, 2.508; $95 \%$ confidence interval, $1.853-10.591 ; \mathrm{P}<0.05)$.

\section{Role of Beclin-1 in the stepwise progression of lung adenocarcinoma}

The expression levels of Beclin-1 gene were $1.69 \pm 0.08$ in NTs, $1.67 \pm 0.10$ in AAH, $1.66 \pm 0.09$ in AIS, $1.52 \pm 0.06$ in MIA, and $1.32 \pm 0.07$ in IA. The expression of Beclin-1 gene was remarkably higher in NTs and precancerous lesions than in IA and MIA. The expression levels of Beclin-1 mRNA in NTs, atypical AAH, and AIS were different, but the difference was not significant between the three, as depicted in Figure 1A. The expression level of Beclin-1 protein was lower in lung cancer tissues than in NTs and precancerous lesions (NTs: $0.63 \pm 0.25, \mathrm{AAH}: 0.59 \pm 0.23$, AIS: $0.58 \pm 0.22$, MIA: $0.26 \pm 0.10$, IA: $0.21 \pm 0.12$ ). As shown in Figure $1 B$ and Figure $1 C$, the relative expression of the difference was statistically significant $(\mathrm{P}<0.05)$. Examples of IHC images of positive Beclin-1 protein are described in Figure 1D. The expression of Beclin-1 in patients with early-stage lung adenocarcinoma (stage $0-\mathrm{I}$ ) was significantly elevated than that in patients with advanced lung adenocarcinoma (stage II-III) $(0.24 \pm 0.11$ vs. $0.17 \pm 0.03$, $\mathrm{P}<0.01)$. The expression of Beclin-1 in the tumor tissues of patients with lung adenocarcinoma who had lymph node metastasis was lower than that in node-negative patients $(\mathrm{P}<0.05)$. The low expression of Beclin-1 predicted a worse survival in patients with IA $(\mathrm{P}<0.05$, Figure 2$)$.

\section{Discussion}

Autophagy is an essential pathway for the degradation of cellular components within the lysosome, and it is highly conserved from yeast to humans (9). Unlike the ubiquitinproteasome system (UPS), which directly degrades multiple regulatory proteins in the cytoplasm and nucleus, the substrates of autophagy are extensive, such as damaged organelles, long-lived proteins, and protein aggregates. 
A
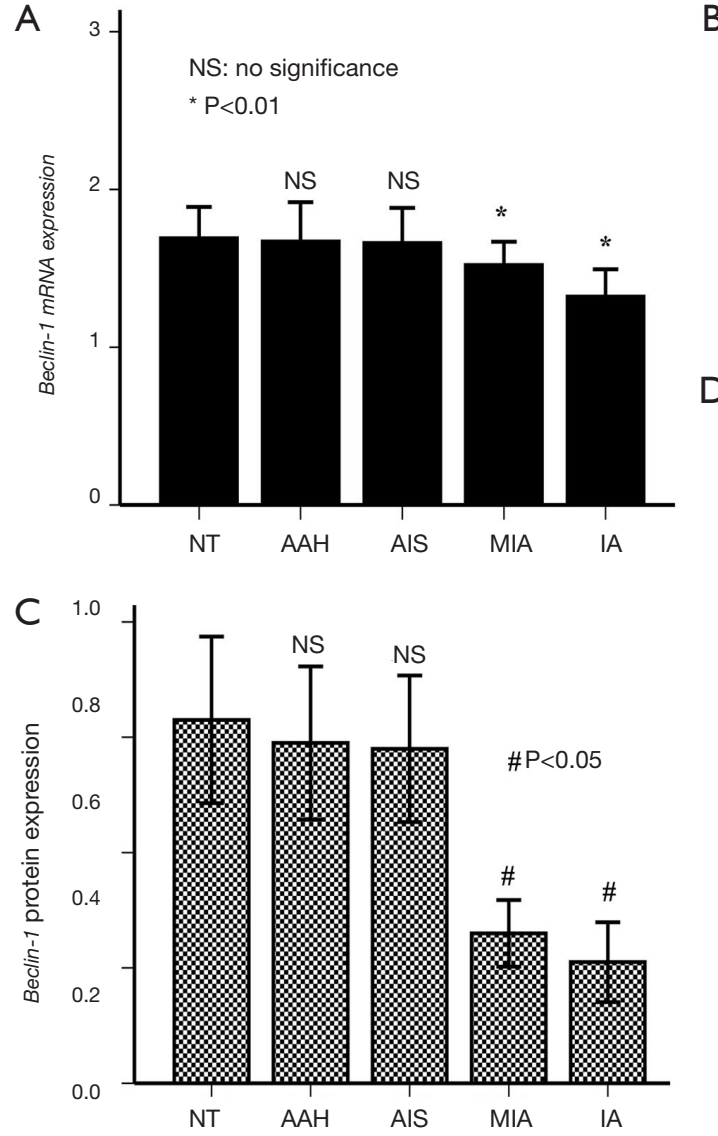

B
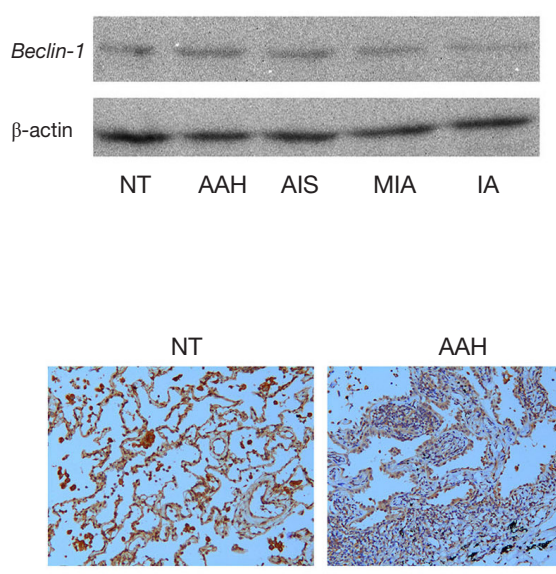

AIS

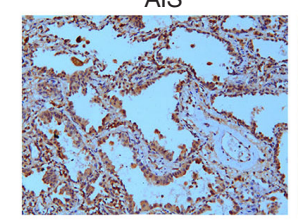

IA

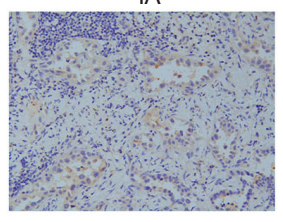

Figure 1 Expression of Beclin-1 mRNA and protein in NT, AAH, AIS, MIA and IA. (A) Beclin-1 mRNA expression; (B) Western blot analysis of Beclin-1 protein expression; (C) densitometry analysis of Beclin-1 protein expression; (D) immunohistochemistry staining of Beclin-1 (original magnification: 100x). NT, lung normal tissues; AAH, atypical adenomatous hyperplasia; AIS, adenocarcinoma in situ; MIA, minimally invasive adenocarcinoma; IA, invasive adenocarcinoma.

Autophagy has been confirmed to be a key regulator of carcinogenesis and may have an influence on the response of therapeutic interventions $(10,11)$. Beclin-1 is an important factor involved in the process of autophagy. Several studies have demonstrated that Beclin-1 was related to a more invasive pathological subtype and poor prognosis (12-15). Moreover, Beclin-1-dependent autophagic function could be suppressed in human cancer by activating AKT $(16,17)$.

Therefore, we hypothesized that autophagy likely play a key role in the genesis and evolution of lung adenocarcinoma. Theoretically, the development of lung adenocarcinoma is a multistep process comprising sequential molecular changes. The IASLC/ATS/ERS introduced the definitions for AAH, AIS, MIA, and IA. So far, no article on the change and significance of Beclin-1 expression during the dynamic evolution of lung adenocarcinoma manifested as GGO has been reported. Meanwhile, with more and more lung adenocarcinoma presenting as GGO are founded, the spectrum of lung cancer changes from advanced to early stage. Therefore, it is very important to study the expression and role of Beclin-1 in lung adenocarcinoma presenting as GGO. Recent studies have reported that AIS may be a precursor of IA. To investigate the role of Beclin-1 in the progression and evolution of lung adenocarcinoma presenting as GGO, we detected the expression of a marker of autophagy Beclin-1, in patients with AAH, AIS, MIA, and IA.

In an earlier study, the expression of Beclin-1 was decreased in lung cancer tissues, and the prognostic impact of Beclin-1 was delineated, indicating its possible role in the pathogenesis of lung cancer (18). Interestingly, another study reported that Beclin-1 expression was inversely 


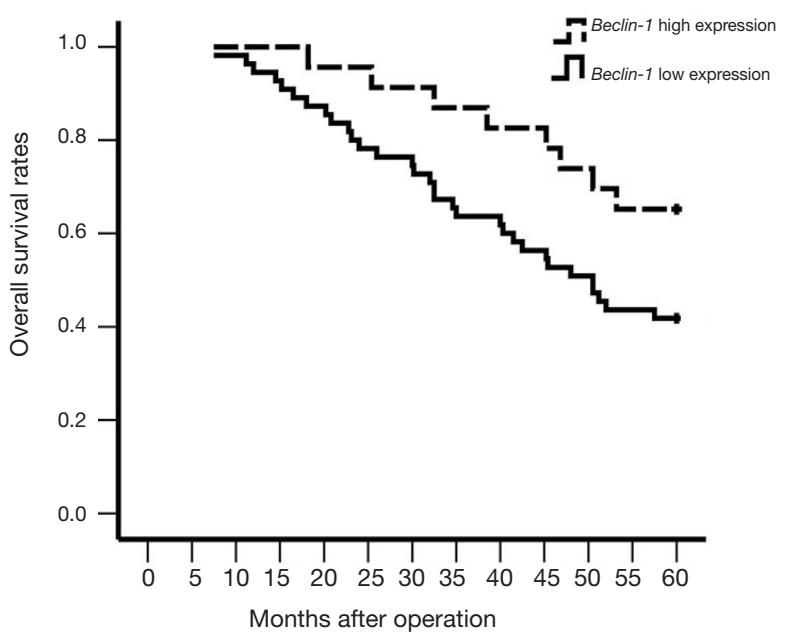

Figure 2 Overall survival of patients with lung adenocarcinoma grouped according to high and low expression levels of Beclin-1.

correlated with the TNM stage of non-small cell lung cancer, but it was not associated with OS (19). Controversies still exist about the role of Beclin-1 in lung cancer. Therefore, we detected the expression levels of mRNA and protein of Beclin-1 in NTs, AAH, AIS, MIA, and IA. We found similar levels of Beclin-1 among NTs, AAH, and AIS. This indicated that autophagy might exert a role in the carcinogenesis of lung adenocarcinoma. Genetic mutation still remains the most important aspect. However, there was a sharp decrease in Beclin-1 expression in patients with MIA and IA compared to that in patients with AIS. The invasive component, excluding the lepidic component, represents the actual malignant component of lung adenocarcinoma. This invasive behavior was characterized by the decrease in Beclin-1 expression. These results indicated that the expression of Beclin-1 could inhibit the progression of the invasive component. The evolution of lung adenocarcinoma was promoted by the decrease in Beclin-1 expression.

Furthermore, the expression of Beclin-1 was low in patients with lymph node metastasis, indicating that Beclin-1 expression might inhibit the metastasis of lung adenocarcinoma. The Kaplan-Meier survival analysis revealed that lower expression of Beclin-1 predicted a significantly worse OS. The multivariate analysis suggested that the expression of Beclin-1 is an independent prognostic factor. When stratified according to the TNM stage in the subgroup analysis, lung adenocarcinoma with low Beclin-1 expression still indicated a worse prognosis. All these results validated that autophagy still plays a role in the progression and metastasis of lung adenocarcinoma presenting as GGO.

The underlying mechanism involved in the suppression of carcinogenesis and progression of lung adenocarcinoma by Beclin-1 is complicated. First, Beclin-1 stabilizes the chromosome structure. Second, autophagy breaks down the damaged cellular components such as organelles. It thus prevents oxidative stress from the damaged organelles (20). Third, Beclin-1 downregulates the proliferation of cells, delays cell cycle progression, and induces autophagy and differentiation (21). Beclin-1 protein may play a significantly different role depending on the type of human cancers. Previous studies have reported complicated results regarding the prognostic impact of Beclin-1 protein expression in different human cancers. Beclin-1 expression was downregulated in liver cancer, ovarian cancer, breast cancer, and gastric cancer, indicating a worse prognostic impact (12-15). However, Beclin-1 expression was upregulated in nasopharyngeal carcinoma and was strongly associated with worse OS and RFS (22). This may be related to the intrinsic properties of the tumor type, and the nature of the therapeutic regimen in different types of malignant tumors. Autophagy may also play a different role in each type of human cancer.

However, there were several limitations in our study. First, the sample size was limited. More valid analysis could be performed only when more samples are included. Second, the basic mechanism of the role of Beclin-1 in the evolution of lung adenocarcinoma was not investigated. We could only infer the function of Beclin-1 from the clinical data. These need to be confirmed by more researches.

\section{Conclusions}

In this study, we observed that Beclin-1 may suppress the carcinogenesis and progression of lung adenocarcinoma presenting as GGO. Further studies are needed to elaborate the underlying mechanism of Beclin-1 expression involved in the evolution of lung adenocarcinoma manifested as GGO.

\section{Acknowledgement}

Funding: This study was supported by Project of Shanghai Jiading District Health Committee (2017KY02).

\section{Footnote}

Reporting Checklist: The authors have completed the 
REMARK reporting checklist. Available at http://dx.doi. org/10.21037/tcr-20-1001

Data Sharing Statement: Available at http://dx.doi. org/10.21037/tcr-20-1001

Conflicts of Interest: All authors have completed the ICMJE uniform disclosure form (available at http://dx.doi. org/10.21037/tcr-20-1001). The authors have no conflicts of interest to declare.

Ethical Statement: The authors are accountable for all aspects of the work in ensuring that questions related to the accuracy or integrity of any part of the work are appropriately investigated and resolved. The study was conducted in accordance with the Declaration of Helsinki (as revised in 2013). The study was approved by Ethics Committee of Ruijin North Hospital (No.2017139) and informed consent was taken from all the patients.

Open Access Statement: This is an Open Access article distributed in accordance with the Creative Commons Attribution-NonCommercial-NoDerivs 4.0 International License (CC BY-NC-ND 4.0), which permits the noncommercial replication and distribution of the article with the strict proviso that no changes or edits are made and the original work is properly cited (including links to both the formal publication through the relevant DOI and the license). See: https://creativecommons.org/licenses/by-nc-nd/4.0/.

\section{References}

1. Lee HY, Choi YL, Lee KS, et al. Pure ground-glass opacity neoplastic lung nodules: histopathology, imaging, and management. AJR Am J Roentgenol 2014;202:W224-33.

2. Travis WD, Brambilla E, Noguchi M, et al. International association for the study of lung cancer/american thoracic society/european respiratory society international multidisciplinary classification of lung adenocarcinoma. J Thorac Oncol 2011;6:244-85.

3. Osarogiagbon RU, Veronesi G, Fang W, et al. Early-Stage NSCLC: Advances in Thoracic Oncology 2018. J Thorac Oncol 2019;14:968-78.

4. Kroemer G, Jaattela M. Lysosomes and autophagy in cell death control. Nat Rev Cancer 2005;5:886-97.

5. Rao S, Yang H, Penninger JM, et al. Autophagy in non-small cell lung carcinogenesis: A positive regulator of antitumor immunosurveillance. Autophagy 2014;10:529-31.

6. White E, Mehnert JM, Chan CS. Autophagy, Metabolism, and Cancer. Clin Cancer Res 2015;21:5037-46.

7. Li YJ, Lei YH, Yao N, et al. Autophagy and multidrug resistance in cancer. Chin J Cancer 2017;36:52.

8. Zhou W, Yue C, Deng J, et al. Autophagic protein Beclin 1 serves as an independent positive prognostic biomarker for non-small cell lung cancer. PLoS One 2013;8:e80338.

9. Ravanan P, Srikumar IF, Talwar P. Autophagy: The spotlight for cellular stress responses. Life Sci 2017; 188:53-67.

10. Nam T, Han JH, Devkota S, et al. Emerging Paradigm of Crosstalk between Autophagy and the UbiquitinProteasome System. Mol Cells 2017;40:897-905.

11. Gomes LR, Menck CFM, Leandro GS. Autophagy Roles in the Modulation of DNA Repair Pathways. Int J Mol Sci 2017; 18:2351.

12. Zhou WH, Tang F, Xu J, et al. Low expression of Beclin 1 , associated with high Bcl-xL, predicts a malignant phenotype and poor prognosis of gastric cancer. Autophagy 2012;8:389-400.

13. Shi YH, Ding ZB, Zhou J, et al. Prognostic significance of Beclin 1-dependent apoptotic activity in hepatocellular carcinoma. Autophagy 2009;5:380-2.

14. Miracco C, Cosci E, Oliveri G, et al. Protein and mRNA expression of autophagy gene Beclin 1 in human brain tumours. Int J Oncol 2007;30:429-36.

15. Tandon M, Othman AH, Ashok V, et al. The role of Runx2 in facilitating autophagy in metastatic breast cancer cells. J Cell Physiol 2018;233:559-71.

16. Wang RC, Wei Y, An Z, et al. Akt-mediated regulation of autophagy and tumorigenesis through Beclin 1 phosphorylation. Science 2012;338:956-9.

17. Chen C, Lu L, Yan S, et al. Autophagy and doxorubicin resistance in cancer. Anticancer Drugs 2018;29:1-9.

18. Jiang ZF, Shao LJ, Wang WM, et al. Decreased expression of Beclin-1 and LC3 in human lung cancer. Mol Biol Rep 2012;39:259-67.

19. Won KY, Kim GY, Lim SJ, et al. Decreased Beclin-1 expression is correlated with the growth of the primary tumor in patients with squamous cell carcinoma and adenocarcinoma of the lung. Hum Pathol 2012;43:62-8.

20. Liang XH, Jackson S, Seaman M, et al. Induction of autophagy and inhibition of tumorigenesis by beclin 1 . Nature 1999;402:672-6. 
21. Zhang L, Gao J, Chen T, et al. Microvesicles Derived from Human Embryonic Neural Stem Cells Inhibit the Apoptosis of HL-1 Cardiomyocytes by Promoting Autophagy and Regulating AKT and mTOR via Transporting HSP-70. Stem Cells Int 2019;2019:6452684.
22. Wan XB, Fan XJ, Chen MY, et al. Elevated Beclin 1 expression is correlated with HIF-1alpha in predicting poor prognosis of nasopharyngeal carcinoma. Autophagy 2010;6:395-404.

Cite this article as: Chen L, Du H, Luo F, Chen X, Li Y, Cheng Q. Beclin-1 expression serves as an important biomarker for carcinogenesis and evolution in lung adenocarcinoma presenting as ground glass opacity. Transl Cancer Res 2020;9(7):4204-4211. doi: 10.21037/tcr-20-1001 